\title{
Chapter 8 \\ Local Assessment of Tokyo: Satoyama and Satoumi - Traditional Landscapes and Management Practices in a Contemporary Urban Environment
}

\author{
Ryo Kohsaka, Wanyu Shih, Osamu Saito, and Satoru Sadohara
}

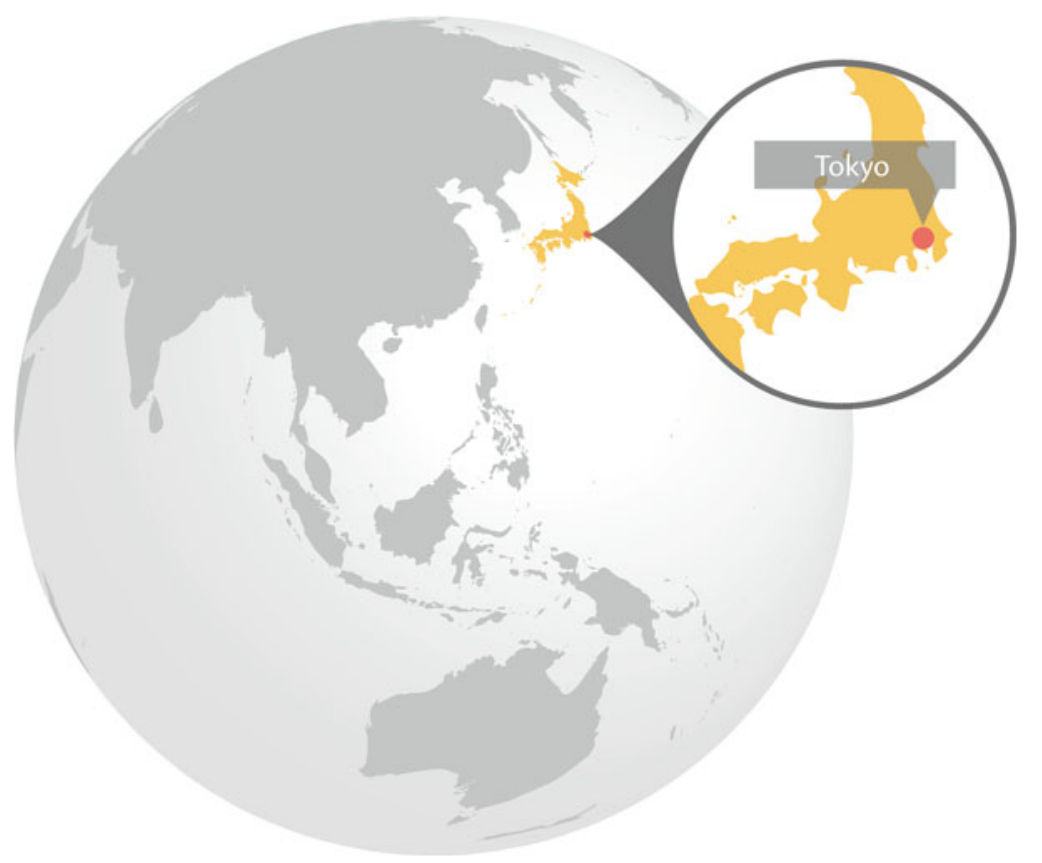

R. Kohsaka ( $\square)$

Graduate School of Human and Socio- Environmental Studies,

Kanazawa University, Kakuma, Kanazawa, Ishikawa 920-1192, Japan

e-mail: kohsaka@hotmail.com

W. Shih

United Nations University-Institute of Advanced Studies (UNU-IAS),

6F International Organizations Center, Pacifico-Yokohama, 1-1-1 Minato Mirai,

Nishi-ku, Yokohama 220-8502, Japan

e-mail: shih@unu.edu

T. Elmqvist et al. (eds.), Urbanization, Biodiversity and Ecosystem Services: Challenges 


\begin{abstract}
This assessment explores the Japanese concepts of satoyama and satoumi (land and coastal), as possible strategies for sustainable management and governance of common urban ecological resources. Satoyama and satoumi are described as landscape types, and management approaches to land and coastal areas that build on a mosaic composition of ecosystem types and their inherent interlinkages. The management practices and the rich biodiversity of the landscapes are thus mutually interdependent. It is acknowledged in the assessment that local governments play a critical role for the management of urban ecosystems and conservation of biodiversity, which is especially important in the face of the unprecedented urban growth currently ongoing globally. This assessment provides an overview of the urbanization trends in Japan, with related challenges to ecosystem provisioning, and the opportunities for sustainable management that a satoyama and satoumi approach can present. Some international examples of ecosystem management that in different ways can inspire transformation of governance structures in Japan to support urban satoyama and satoumi are highlighted.
\end{abstract}

\title{
Key Findings
}

- Satoyama and satoumi landscapes are biodiversity-rich landscapes in peri-urban to rural areas, and contain a mosaic of ecosystems sustained by human management.

- Extensive rural-to-urban migration and expanding cities in Japan have caused a decrease of use of the natural resources, resulting in a degradation of the quality and quantity of satoumi and satoyama landscapes.

- Implementing satoyama and satoumi in urban areas can support cultural, provisioning and regulating ecosystem services, thereby also well-being for humans and ecosystems alike.

- Current urban green space planning policies do not sufficiently take the satoyama and satoumi concepts into account, and have mostly focused on national and regional levels, whereas support from both local governments and communities is key for implementation and long-term management.

- Two elements are argued to support implementation and long-term management of urban satoyama and satoumi: an increased involvement of local communities in the policy-making and management of the urban green areas, and incentives to make urban satoyama and satoumi financially profitable.

O. Saito

Institute for Sustainability and Peace (UNU-ISP), United Nations University, 5-53-70 Jingumae, Shibuya-ku, Tokyo 150-8925, Japan

The University of Tokyo, Tokyo, Japan

e-mail: saito@unu.edu

S. Sadohara

Graduate School of Urban Innovation, Yokohama National University,

79-7 Tokiwadai, Hodogaya-ku, Yokohama 240-8501, Japan

e-mail: sadohara@ynu.ac.jp 


\subsection{Introduction}

The values of urban ecosystems for human well-being are increasingly recognized in research, although they may not always be recognized by the general public (see for example Mougeot 2006; Vandruff et al. 1995; Bolund and Hunhammar 1999; Berkowitz et al. 2003) (see also Chaps. 11 and 27). In Japan, the interdependence between traditional activities and natural environments is the foundation of Japanese culture. Following this, cultural services such as educational and social services are increasingly appreciated by urban residents.

Satoyama (land) and satoumi (coastal) are Japanese concepts that refer to long-standing management traditions based on the symbiotic interaction between ecosystems and humans (Duraiappah et al. 2012). Satoyama and satoumi landscapes are defined as "a dynamic mosaic of managed socio-ecological systems producing a bundle of ecosystem services for human well-being" (Saito and Shibata 2012, p. 26).

The four primary characteristics of these landscapes are: (1) satoyama is a mosaic of both terrestrial and aquatic ecosystems comprised of woodlands, plantation, grasslands, farmlands, pasture, irrigation ponds and canals, with an emphasis on the terrestrial ecosystems; (2) satoumi refers to aquatic ecosystems and is a mosaic of both terrestrial and aquatic ecosystems comprised of seashores, rocky shores, tidal flats, coral reefs, and seaweed/grass beds; (3) satoyama and satoumi landscapes are managed with a mix of traditional knowledge and modern science; and (4) biodiversity is a key element for the resilience and functioning of satoyama and satoumi landscapes.

Satoyama and satoumi are primarily found in rural and peri-urban areas and satoyama alone is estimated to cover $40 \%$ of Japan's total landmass, in some prefectures reaching up to $58 \%$ (Ministry of the Environment, Japan 2001). The current trend is going towards degradation in quality and quantity of the landscapes: satoyama primarily due to land usage change, i.e., conversion of farmland and forests to built-up urban areas; and satoumi due to overfishing, pollution, and changes to the physical environment caused by intentional development or unintentional human activities of coastal areas, such as land reclamation, port constructions and coastal embankments. However, transferring satoyama and satoumi to cities holds the potential to maintain these landscape management approaches, while at the same time provide effective ways to meet some of the challenges of the growing cities. For example, ensuring food security and production of fresh water can be particularly important in developing countries where urbanization rates are high.

The transition of satoyama and satoumi landscapes to urban areas is, however, faced with some key obstacles: (1) a lack of awareness of the potential social and ecological benefits of satoyama and satoumi; (2) a lack of legislative and policy support to control the urban expansion, conserve satoyama and satoumi in the new urban areas, and implement new such landscapes in the already built-up environment; (3) a lack of technological, human, and financial resources for implementation; and (4) a lack of coordination across institutional levels (JSSA 2010a; Ministry of the Environment, Japan 2012). 
The next section provides an overview of urbanization and its effects on biodiversity and ecosystem services in Japan, focusing on satoyama and satoumi landscapes located in or nearby urban areas. The text will then explore some approaches to biodiversity governance that manage the changes associated with urbanization around the world, with the aim to assess possible ways to strengthen implementation of an urban satoyama and satoumi approach.

\subsection{Urbanization, Ecosystem Services and Biodiversity: Scenarios and Trends in Japanese Cities}

The intensive growth of industries during the rapid economic growth period from the late 1950s to the early 1970s in Japan created employment opportunities, led to population concentration, and ultimately urbanization. Particularly the economic globalization of the last decades of the 1900s, created urban concentrations and eventually metropolises on the Pacific coastal zone of Japan, such as Kobe, Osaka, Nagoya, and Tokyo.

The rapidly expanding cities resulted in conversion of farmland to urban land, first along the coastline, then into forests and in-land (Himiyama 2004). In the process, people's traditional ways of making their livelihoods significantly changed (Okuro et al. 2012). In the rural areas, modernization and mechanization of agriculture created monocultures, which decreased biodiversity (Okuro et al. 2012). At the same time, forestry and fisheries collapsed due to the expanding cities and the import of timber and seafood.

Together with the degradation of satoyama management practices, the capacity of the ecosystems to provide crucial services such as nutrient cycling, food and timber production, water retention, and water purification has decreased. In addition to agricultural production, the change in satoyama also affected the ecosystem composition and local culture. From the 1990s, an ageing rural population and shrinking working population within agriculture, forestry and fisheries have both become increasingly apparent nationwide.

At the same time, the changes in land cover from natural landscapes to built-up areas also create challenges for the service providing ecosystems. The population concentration in the Tokyo metropolitan area, which includes the neighboring prefectures around Tokyo (i.e., Kanagawa, Saitama, and Chiba), consequently forms the world's largest city zone with over 30 million people. The Kanto region, i.e., the Tokyo metropolitan area and its three neighboring prefectures: Gunma, Tochigi and Ibaraki, has been exposed to the most rapid and extensive urban development and population growth in Japan. When the urban population increase was the most dramatic, i.e., in the 1960s until the 1980s, the forest cover in the region decreased by $4.3 \%\left(618 \mathrm{~km}^{2}\right)$ (Saito 2004).

Japan is today facing several changes within cities as well as in the peri-urban areas. Some of the largest cities are expected to decrease in population size, as the 
country's overall population began to decline in 2005. It is predicted that the urban populations will decrease rapidly and at the same time the mean age will increase, especially in big cities (National Institute of Population and Social Security Research 2012). Securing a workforce to maintain the production of ecosystem services has become difficult not only in rural satoyama but also in satoyama located in or close to the metropolitan areas, (i.e., the cities and the neighboring prefectures).

However, overconcentration in the center of cities is expected to continue in some metropolises like the Tokyo metropolitan area. At the same time, population numbers in the peri-urban areas around the city centers have dwindled due to depopulation and migration from the crowded city centers to peripheral areas. As the population in the city centers decrease, the urban sprawl increases.

\subsubsection{Changes and Feedbacks in Ecosystems}

The decrease of land and marine resources management in Japan, has caused a degradation of products provided by satoyama and satoumi such as timber, crops and fish. This, in turn, has caused degradation of other types of ecosystem services including supporting, regulating, and cultural services (Duraiappah et al. 2012).

The changes in ecosystem services provisioning in areas outside and around metropolitan areas can come to increase the effects of other expected changes, as they reduce the natural disaster buffer capacities. Climate changes are expected to increase the risks of extreme weather phenomena and related disasters in Japan, and the metropolitan areas like Tokyo and Osaka are expected to experience the biggest changes. Heat island effects are already advancing, and extreme weather phenomena such as guerrilla downpours and intense heat, occur frequently.

As the quality of the rural land decreases, so does the biodiversity, and thereby also the resource base that supports the cities with bundles of ecosystem services. These changes have already led to increased frequencies of floods and threats to the availability of indispensable high-quality fresh water of upstream regions.

Satoyama landscapes in peri-urban areas have been faced with new challenges including fragmentation of landscapes, loss of mosaic land use, increase of alien species like Solidago altissima and common raccoon (Procyon lotor), and loss of traditional knowledge to manage the landscape. The loss of traditional knowledge is particularly significant. In a study by Shimada et al. (2008), four past and present management models of typical secondary woodlands in satoyama near Tokyo were identified: traditional management, non-traditional management, ad hoc management, and no management. They found that traditional management maintained relatively higher species richness with little variation between surveyed plots, compared to non-traditional management models.

Japan, as with many countries around the world, imports a substantial proportion of its consumed resources. This pattern of reliance on resources from around 
the world, to a large extent driven by demands by urban dwellers, has strongly contributed to degradation of ecosystem services in rural areas globally, such as soil loss, ground drainage, reduced amounts of carbon storage because of deforestation, and global biodiversity loss. The role of urban areas as strong contributors to global environmental problems needs to be acknowledged. It is, however, also crucial to acknowledge and realize the potential of cities to act as areas for adaptation and mitigation strategies and as hubs for innovations (Chap. 33).

\subsection{Governance and Institutions}

Although policies and ordinances to conserve and maintain urban green spaces for multiple ecosystem services, such as food security, local climate regulation, and biodiversity conservation exist, they are not necessarily associated with the concept of satoyama and satoumi. This part of the assessment intends to address this gap and use existing examples from other countries to facilitate the discussion of how good governance of satoyama and satoumi at a local or city level in Japan may be designed.

An assessment aimed to provide policy-makers with scientifically credible information on the values of ecosystem services provided by satoyama and satoumi for economic and human development, the Japan Satoyama Satoumi Assessment (JSSA), was initiated in Japan in 2006. The final report was published in 2012 (Duraiappah et al. 2012). The JSSA primarily focused on Japan on a national and regional scale, but also included a local urban and peri-urban scale. In addition to discussing changes in ecosystem services, the JSSA also covered institutional mechanisms, socio-economic challenges, public participation, and associated appraisal of biodiversity governance.

The JSSA shows how satoyama and satoumi emphasize sustainable use of ecosystems in order to support the provisioning of ecosystem services for human well-being. However, in practice, the general ecosystem management and governance discussions have thus far mostly focused on regional and local levels, whereas the discussions on satoyama and satoumi in the JSSA focused on national and subnational levels. There is thus a need to develop strategies that address local management and governance of urban satoyama and satoumi in Japan.

Based on the findings in the JSSA, four aspects that may facilitate the development of local governance in support of urban satoyama and satoumi are discussed: (1) effectiveness of biodiversity policy; (2) coordination between development and biodiversity and ecosystem services conservation; (3) available financial mechanisms to support long term implementation; and (4) capability to build up partnerships and encourage participation. Furthering the discussion on strategies that may improve the dynamic balance between societies and ecosystems in cities, this assessment draws upon examples from Nagoya, Japan; eThekwini/Durban, South Africa; and the UK (Europe). 


\subsubsection{Strategies for Effective Biodiversity Conservation Policies}

Biodiversity conservation is a national goal in Japan as clearly defined in the Japanese National Biodiversity Strategies and Action Plan (NBSAP) (the Fifth Edition has been recently launched on September 2012). The Japanese NBSAP has explicitly taken satoyama and satoumi and urban green spaces into account but separately of each other. However, satoyama and satoumi landscapes, whether rural or urban, are thereby still treated as separate from urban nature and lack conceptual alignment in policies. For example, the mosaic characteristics of forests and agricultural lands make it difficult to categorize the satoyama into one category of land classification. Local ordinances have been proposed by prefectures and cities in Japan to promote conservation, regeneration and utilization of satoyama since 2000 (Takahashi et al. 2012). However, regulations and policies at a local level tend to favor economic growth and development before protection of existing urban satoyama and satoumi. In this context, the loss of natural and semi-natural areas, which are the basis of satoyama and satoumi, is inevitable without effective legal or non-legal binding instruments aimed at protection of the natural landscapes.

Following a segmented governance structure, national policies and laws in Japan are largely separated from the local governance and thus provide little support for satoumi and satoyama, although it is recognized in the national guidelines that an integrated approach is needed for managing such a system in a sustainable manner (Takahashi et al. 2012). Legal responses have been respectively developed to address the need of sustaining satoyama and satoumi landscape at national levels. The conservation of satoyama in Japan is promoted under the newly established Act on the Promotion of Conservation for Biodiversity Activities through the Cooperation among Regional Diversified Actors (enforced in 2011). However, the development of specific legal strategies for conservation of satoumi and by that marine biodiversity, is still only in the early stages. Management of coastal areas is marked by a highly complex web of a wide range of stakeholders from fisheries, construction, nature conservation, and recreation sectors, among others.

An international and seemingly promising method is the Strategic Environmental Assessment (SEA), which since 2004 is mandatory under the SEA Directive for the member states of the European Union to conduct in selected plans and programs. SEA originates from the National Environmental Policy Act (NEPA) of the USA in 1970, and has since mostly been developed and implemented in European and North American countries (Dalal-Clayton and Sadler 2005). SEA is designed to evaluate the possible accumulative environmental consequences of proposed policies and plans, and thus ensure already in the early stages of decision making that biodiversity support is considered (ICLEI 2010). Although SEA systems vary considerably between countries or even cases, the concept has rapidly evolved and been applied to public plans and programs, such as land use, transport, energy, waste and agriculture, to support sustainable development. 
In the past decades, the concept of SEA has been introduced in several East Asian countries, including China, South Korea, Taiwan, and Japan. As in many East Asian countries, the applications in Japan remain limited, but some SEA components have already been implemented as local governments use the SEA to screen environment-related plans and programmes (Dalal-Clayton and Sadler 2005). SEA can provide an opportunity to include satoyama and satoumi approaches into development processes, if the SEA system would be further developed and adapted to a Japanese social-ecological context. For example, the importance of using traditional knowledge for environmental management, as well as the interaction between human maintenance activities and their consequent ecosystem functioning will have to be highlighted and valued in the SEA.

\subsubsection{Development That Supports Biodiversity and Ecosystem Services Conservation}

Laws, regulations, and policies proposed for green space conservation and the sustainable use of natural resources have shown to some extent to contribute to the maintenance of satoyama and satoumi landscapes (Takahashi et al. 2012). However, implementation and long-term management of satoyama and satoumi in and around cities is complicated by the increasing competition for land in Japan. One of the key challenges to make satoyama and satoumi an integral part of the urban landscape is thus to find strategies that can balance land-use for financial prosperity, with the implementation and conservation of satoyama and satoumi. Another key challenge is to design plans and strategies of which satoyama and satoumi are integral parts.

Although plans and strategies that aimed to include biodiversity and ecosystem services in urban development have been designed, for example a Master Plan for Greenery, their relation to urban satoyama and satoumi is not specified. Their full potential to support satoyama and satoumi is thus still to be realized.

One way to meet the limitations of current plans may be to design more comprehensive development plans that systematically coordinate biodiversity protection and development initiatives. Taking the UK's planning system as an example, the Biodiversity Supplementary Planning Documents (BSPDs) are developed in conjunction with local development documents. A BSPD provides explicit guidance to actors such as developers, households and planners on protecting, creating and improving biodiversity during the development process (SCDC 2012). Another systematic planning approach that facilitates biodiversity and development coordination is the South African Integrated Development Plans (IDPs) which are mandatory for all local authorities in South Africa to prepare and continue over 5 years. By aiming to coordinate the work between different sectors, such as housing, environments and transportation, the IDPs can provide a cross-sectoral planning mechanism that integrates development with conservation of biodiversity and ecosystem services (eThekwini Municipality 2007). These planning systems take biodiversity 
and ecosystem services into account at the early stage of local development and stand better chances to influence and to be compatible with other sectoral plans.

The BSPDs and the IDPs represent two top-down planning measures that might facilitate the integration of satoyama and satoumi proposition throughout existing development frameworks and influence sectoral plans. However, both of these measures are relatively new and it is too early to conclude which measure is more applicable in the Japanese context. More studies to address the characteristics of Japanese planning and institutional systems are needed.

In an interesting bottom-up development, on the other hand, a revival movement of satoyama landscapes since the late 1980s, has increasingly drawn public attention and interest to nature conservation and protection. This movement has been especially active in municipalities containing urban satoyama and satoumi, with several tens of thousands municipal inhabitants, located within approximately $50 \mathrm{~km}$ from urban centers (Saito 2005). The activities focus mostly on the values of cultural services such as education and recreation. This hints to that supporting cross-scale planning mechanisms where urban inhabitants are involved in planning and management, can promote urban satoyama and satoumi on several levels.

\subsubsection{Available Financial Mechanisms to Support Long-Term Implementation}

International initiatives to give ecosystem services monetary values have for example estimated the urban plantations in Canberra, Australia, at a combined energy reduction, pollution mitigation and carbon sequestration value of US\$20-67 million during the period 2008-2012 (Brack 2002). If satoyama and satoumi can credibly be given a monetary value, this can be an incentive to support the conservation of satoyama and satoumi landscapes in Japan (e.g., TEEB 2011).

Several economic interventions have already been proposed by Japanese local governments to mitigate or reverse the decline in satoyama and satoumi. The interventions include taxation of illegal industrial waste dumping, encouragement of the use of biomass energy from thinning woods of satoyama, and direct payments for stewardship, i.e. management aimed to maintain and conserve the rural natural resources. The implementation of the incentives has, however, been limited due to the decreasing production value from agricultural, forestry and fishery activities (Takahashi et al. 2012).

Voluntary involvement plays an increasingly large role for maintenance of satoyama and satoumi, such as payment for ecosystem services, stewardship sharing with citizens, and certification systems for products (Takahashi et al. 2012). A case in point is the Greenification Certificate System initiated in Nagoya, Japan in 2008 (cf. Kohsaka 2010). Under the overall framework of the System of Greening Areas, the Greenification Certificate System serves as a voluntary tool by which private landowners receive lower interest rates on loans from local banks, while conserving or creating green spaces when they develop their land. This experimental tool is 
expected to encourage more green spaces, including trees, green facades and green roofs, in private owned properties and might be useful for enhancing biodiversity in densely urbanized cities.

\subsubsection{Capacities to Build Partnerships and Encourage Participation}

As human activities play a vital role in the management of satoyama and satoumi, participation from citizens, local non-profit organisations (NPOs) and nongovernmental organisations (NGOs) should be acknowledged by decision makers and in planning as a key component for achieving sustainable management of urban satoyama and satoumi. Involving a wide range of partners, such as government agencies, academia, conservation groups, local businesses, amateur naturalists and private corporations, can play a key role in defining priorities in satoyama and satoumi management and sustain it over time.

One international example of a successful public-private partnership that may serve as inspiration for cities in Japan, is the Buffelsdraai Community Reforestation Project in South Africa. The eThekwini municipality and the Wildlands Conservation Trust together engage local communities to create tree nurseries at their homes and provide tree seedlings for reforestation. By selling seedlings, participants receive credit notes to exchange of food, basic goods and school fees at regular tree stores in participating communities (eThekwini Municipality 2012).

In Japan, Kawasaki City developed a Conservation Management Plan to engage citizens in stewardship activities of designated conservation areas and to reduce the conservation burden in terms of labor and expenditure on landowners. The city also builds a partnership with universities to encourage research on urban ecosystem conservation areas and to open up a dialogue between academics, policy-makers and managers. The capacity to build such cross-level and cross-sectoral partnerships where local inhabitants actively participate in the management of the urban green areas can prove to be critical in terms of creating and sustaining urban satoyama and satoumi (Chap. 27).

\subsection{Concluding Remarks}

Although satoyama and satoumi are Japanese terms, these landscapes of mosaic ecosystems where human-nature interaction is central are not unique to Japan. Such landscapes are found throughout many regions of the world, though the terminology and managerial philosophy might vary from one area to the other (Duraiappah et al. 2012).

This text has assessed satoyama and satoumi landscapes as possible strategies to support and urban development that builds on conservation of urban ecosystems. 
The concepts originally referred to specific landscape types as well as management structures on a regional scale in Japan. As cities are expanding on traditional satoyama and satoumi landscapes, however, the concepts increasingly refer to existing or planned areas in or around cities.

Management of satoyama and satoumi has traditionally built on involvement by entire communities. Translated to urban areas, satoyama and satoumi may thus provide support for inclusive, multi-level management structures as well as management of landscapes. Including the local inhabitants and their ecological knowledge can be a means of supporting and maximizing the ecosystem functioning, as well as ensuring long-term management.

However, several factors challenge both conservation of existing satoyama and satoumi areas and successful implementation of urban satoyama and satoumi landscapes. These challenges include weak legislation where the existing regulations give insufficient support for ecosystem conservation in general, and in addition lacks integration of satoyama and satoumi aspects. The increasingly stiff competition for land in Japanese cities, and even more so in cities in the most rapidly urbanizing countries in the world, pushes land prices up and challenges green area conservation. Finally, climate changes affect the prerequisites for native species to exist, and their inherent balance, thus also affecting entire ecosystems. To highlight possible ways to meet the challenges, the assessment has shown through international examples that entail satoyama and satoumi elements, where local initiatives have contributed to successful ecosystem management.

Although many green initiatives exist in cities such as Yokohama (JSSA 2010b; Sadohara et al. 2011), Nagoya (Nagoya City 2010) and Kanazawa (Ishikawa Prefecture 2011; UNU-IAS 2011), in the long term these obstacles require changes at multiple levels, from governments to societies. Local governments will play a critical role in initiating appropriate changes to favor biodiversity and ecosystem health in the course of urban development. Therefore, a proactive assessment framework that directs local governments to address problems and to monitor performance is needed for further development.

Open Access This chapter is distributed under the terms of the Creative Commons Attribution Noncommercial License, which permits any noncommercial use, distribution, and reproduction in any medium, provided the original author(s) and source are credited.

\section{References}

Berkowitz, A. R., Nilon, C. H., \& Hollweg, K. S. (Eds.). (2003). Understanding urban ecosystems: A new frontier for science and education. New York: Springer.

Bolund, P., \& Hunhammar, S. (1999). Ecosystem services in urban areas. Ecological Economics, 29, 293-301.

Brack, C. L. (2002). Pollution mitigation and carbon sequestration by an urban forest. Environmental Pollution, 16, 195-200.

Dalal-Clayton, B., \& Sadler, B. (2005). Strategic environmental assessment: A sourcebook and reference guide to international experience. London: Earthscan. 
Duraiappah, A. K., Nakamura, K., Takeuchi, K., Watanabe, M., \& Nishi, M. (Ed.). (2012). Satoyama-Satoumi ecosystems and human well-being: Socio-ecological production landscapes of Japan. Tokyo: United Nations Press, 480 pp.

eThekwini Municipality. (2007). LAB biodiversity report. Durban: EThekwini Municipality.

eThekwini Municipality. (2012). Buffelsdraai community reforestation project. Available online at http://www.durban.gov.za/City_Services/development_planning_management/environmental_planning_climate_protection/Projects/Pages/Buffelsdraai-Community-ReforestationProject.aspx. Accessed 18 May 2012

Himiyama, Y. (2004). The trend, mechanism and impact of urban expansion in Japan. Reports of the Taisetsuzan Institute of Science (Hokkaido University of Education), 38, 53-60.

ICLEI - Local Governments for Sustainability. (2010). Local action for biodiversity guidebook: Biodiversity management for local Governments (M. T. Loaros \& F. E. Jones, Eds.).

Ishikawa Prefecture. (2011). Ishikawa biodiversity strategy vision (Written in Japanese).

Japan Satoyama Satoumi Assessment (JSSA). (2010a). Satoyama-Satoumi ecosystems and human well-being: Socio-ecological production landscapes of Japan - Summary for decision makers. Tokyo: United Nations University.

Japan Satoyama Satoumi Assessment (JSSA). (2010b). Satoyama and Satoumi: Socio-ecological production landscapes in Japan - Experiences and lessons from Kanto-chubu cluster-Kantochubu Cluster. Tokyo: United Nations University.

Kohsaka, R. (2010). Economics and the convention on biodiversity: Financial incentives for encouraging biodiversity in Nagoya. In N. Muller, P. Werner, Urban, \& G. Kelcey (Eds.), Biodiversity and design (pp. 593-607). West Sussex: Wiley-Blackwell.

Ministry of the Environment, Japan. (2001). Study on Satochi-satoyama in Japan: Interim report. Tokyo: Ministry of the Environment, Japan (Written in Japanese). Available at: http://www. env.go.jp/nature/satoyama/chukan.html

Ministry of the Environment, Japan. (2012). The National biodiversity strategy of Japan 20122020: Living in harmony with nature. Tokyo.

Mougeot, L. J. A. (2006). Growing better cities: Urban agriculture for sustainable development. International Development Research Center (IDRC), Ottawa.

Nagoya City. (2010). Biodiversity 2050 Nagoya strategy (Written in Japanese).

National Institute of Population and Social Security Research. (2012). Population projections for Japan (January 2012): 2011 to 2060. Available online at http://www.ipss.go.jp/site-ad/index english/esuikei/gh2401e.asp. Accessed 7 Feb 2013.

Okuro, T., Yumoto, T., Matsuda, H., \& Hayashi, N. (2012). What are the key drivers of change and current status of satoyama and satoumi? In A. K. Duraiappah, K. Nakamura, K. Takeuchi, M. Watanabe, \& M. Nishi (Eds.), Satoyama-Satoumi ecosystems and human well-being: Socioecological production landscapes of Japan (pp. 60-124). Tokyo: United Nations Press.

Sadohara, S., Koike, F., Kada, R., \& Sato, Y. (2011). SATOYAMA revitalization - Challenges of Yokohama City and Kanagawa Prefecture, Japan-, Soshinsha (written in Japanese).

Saito, O. (2004). Quantitative and special changes in woodland vegetation for the last 40 years in the Kanto region, Japan. Theory and Applications of GIS, 12(2), 146-154 (In Japanese with English abstract).

Saito, O. (2005). Characteristics of Satoyama interactive activities. In Nature Conservation Society of Japan (Ed.), Satoyama nature from the ecological perspective and the protection (pp. 195-201). Tokyo: Kohdansha.

Saito, O., \& Shibata, H. (2012). Satoyama and Satoumi, and ecosystem services: A conceptual framework. In A. K. Duraiappah, K. Nakamura, K. Takeuchi, M. Watanabe, \& M. Nishi (Eds.), Satoyama-Satoumi ecosystems and human well-being: Socio-ecological production landscapes of Japan (pp. 17-59). Tokyo: United Nations Press.

Shimada, K., Katsuki, T., Iwamoto, K., \& Saito, O. (2008). Management effects on the community structure and species richness of secondary Quercus serrata -Q.acutissima woodland in the southwest Tama area, Tokyo, Japan. Vegetation Science, 25, 1-12 (In Japanese with English abstract). 
South Cambridgeshire District Council. (2012). Biodiversity SPD. Available online at http://www. scambs.gov.uk/content/biodiversity-spd. Accessed 2 Sep 2013.

Takahashi, T., Isozaki, H., Oikawa, H., Oyama, K., \& Kunimitsu, Y. (2012). What and how effective have the main responses to address changes in satoyama and satoumi been? In A. K. Duraiappah, K. Nakamura, K. Takeuchi, M. Watanabe, \& M. Nishi (Eds.), Satoyama-Satoumi ecosystems and human well-being: Socio-ecological production landscapes of Japan (pp. 155-188). Tokyo: United Nations Press.

The Economics of Ecosystems and Biodiversity (TEEB). (2011). TEEB manual for cities: Ecosystem services in urban management. London: Earthscan.

United Nations University Institute for Advanced Study (UNU-IAS). (2011). Biodiversity in Kanazawa (69pp.). Tokyo: UNU.

Vandruff, L. W., Leedy, D. L., \& Stearns, F. W. (1995). Urban wildlife and human well-being. In H. Sukopp, M. Numata, \& A. Huber (Eds.), Urban ecology as the basis for urban planning (pp. 203-211). Amsterdam: SPB Academic publishing. 